\title{
Association between allostatic load and health behaviours: a latent class approach
}

\author{
Sarah N Forrester, ${ }^{\oplus} 1$ Jeannie-Marie Leoutsakos, ${ }^{2}$ Joseph J Gallo, ${ }^{3}$ Roland J Thorpe, ${ }^{4}$ \\ Teresa E Seeman ${ }^{5}$
}

${ }^{1}$ Quantitative Health Sciences, University of Massachusetts Medical School, Worcester, Massachusetts, USA ${ }^{2}$ Johns Hopkins University School of Medicine, Department of Neuropsychiatry, Baltimore, Maryland, USA

${ }^{3}$ Department of Mental Health, Johns Hopkins University Bloomberg School of Public Health, Baltimore, Maryland, USA

${ }^{4}$ Health Behavior and Society, Johns Hopkins Bloomberg School of Public Health, Baltimore, Maryland, USA ${ }^{5}$ Los Angeles Geffen School of Medicine, University of California, Los Angeles, California, USA

\section{Correspondence to} Dr Sarah N Forrester, Quantitative Health Sciences Department, University of Massachusetts School of Medicine, Worcester, MA 01605, USA; sarah.forrester@ umassmed.edu

Received 4 July 2018 Revised 30 November 2018 Accepted 11 January 2019 Published Online First 30 January 2019

\section{Check for updates}

(C) Author(s) (or their employer(s)) 2019. No commercial re-use. See rights and permissions. Published by BMJ.

To cite: Forrester SN, Leoutsakos J-M, Gallo JJ, et al. J Epidemiol Community Health 2019:73:340-345.

\begin{abstract}
Background Allostatic load (AL) has been characterised in many ways throughout the literature; however, its relationship to health behaviours has only been studied in limited populations. We aimed to uncover qualitative patterns of biological indicators in $\mathrm{AL}$ and determine if those patterns were associated with certain health behaviours.

Methods We conducted latent class analysis using biological indicators from a multiethnic population. We fit latent class regression of class on health behaviours (smoking, poor diet, physical activity and alcohol use) to measure the association between each latent class of $\mathrm{AL}$ and each health behaviour.

Results Four classes, 'Metabolic+Cholesterol, 'Blood Pressure', 'Metabolic+Blood Pressure' and 'Low', were found in the sample. Latent class regression showed that physical activity and alcohol use were significantly associated with the 'Metabolic+Blood Pressure' class. Conclusion Less physical activity was required to improve AL than was previously found. Low to moderate alcohol use was beneficial for lower AL. Implications of the amount of physical activity necessary to lower $A L$ is discussed.
\end{abstract}

\section{INTRODUCTION}

Allostatic load (AL) is the cumulative biological toll on the body due to prolonged chronic stress or stress that is constant and continues over an extended period of time. ${ }^{1}$ Although allostasis is a normal physiological adaptation mechanism, which allows bodily systems to react to threat and stress when necessary, chronic stress can result in overactivation of, for example, the sympathetic nervous system and hypothalamic-pituitary-adrenal axis. ${ }^{1}$ Conceptually, $\mathrm{AL}$ arises in response to whatever an individual's brain perceives as stressful so, in theory, the actual stressors that increase a person's AL are highly individualised. $^{2}$ In recent years, AL has become a popular mechanism for studying the toll of stress on multiple bodily systems. Most biological indicators that are commonly used in the construction of AL (eg, blood pressure, haemoglobin $\mathrm{A} 1 \mathrm{C}$ (HbA1c), cholesterol and body mass index) are associated with lifestyle and behaviour (eg, cigarette smoking is associated with cardiovascular disease), ${ }^{3}$ but how health behaviours and lifestyle factors are associated with $\mathrm{AL}$ as a whole is poorly understood. Recently, a few studies have shown an association between AL and poor $\operatorname{diet}^{4}$ and between AL and physical activity ${ }^{5-7}$ in Puerto Rican and Mexican populations. However, more often than not, these behaviours are simply included as control variables in a model testing the relationship between AL and another outcome. ${ }^{8-12}$ This lack of attention to behavioural factors associated with AL is why we choose to focus on diet, exercise, smoking and alcohol use. These are very common behavioural factors and thus would be expected to affect a larger number of people in the population.

Among the challenges of using AL is gaining a better understanding of the types of stress that can increase it and whether lifestyle factors can affect AL in the way that psychological stress does. ${ }^{13}$ Making health behaviours the focus of the analysis rather than just including them as confounders may allow us to better understand how these modifiable risk factors are associated with AL as a construct. Measurement of AL has been largely dependent on the biological measures available in study data. To an extent, the markers we chose were dependent on availability, but we specifically chose to use markers that would represent primary mediators of AL (eg, cortisol, norepinephrine and epinephrine) as well as secondary outcomes of AL that are commonly affected by behavioural choices (eg, blood pressure, cholesterol and waist-to-hip ratio [WHR]) so that the results would be useful both clinically and to further AL research. We used latent class analysis (LCA) in order to determine if there were clusters of AL indicators within the sample and to explore the relationship between latent classes of $\mathrm{AL}$ and common health behaviours such as smoking, alcohol use, diet and physical activity. We were interested in how health behaviours were associated with $\mathrm{AL}$ as a whole and how specific health behaviours might influence components of the AL construct. The main goal of this research was to uncover possible qualitative differences in patterns of biological indicators of AL that may not be evident when the indicators are simply added together to create a total score. We used LCA because it allowed us to explore qualitative differences in AL indicators, and we hypothesised that qualitatively different classes of AL would be found and that health behaviours would be associated with AL patterns.

\section{METHODS}

\section{Participants}

The sample was derived from the Stress substudy of the Multiethnic Study of Atherosclerosis (MESA). Details of the parent study can be found elsewhere. ${ }^{14}$ Briefly, 6814 participants, free of cardiovascular disease, who reported their race/ ethnicity as white, African-American, Hispanic or Asian were recruited from six study centres in various states throughout the USA. The Internal 
Review Board for each study site approved the protocols, and all participants gave written informed consent. There were five examinations total between 2000 and 2012. The MESA Stress ancillary study was conducted between 2004 and 2006 in conjunction with exams 3 and 4 of the parent study. One thousand and two participants were enrolled in MESA Stress from the New York and Los Angeles study sites. Participants were enrolled in the order in which they came for their follow-up exams until about 500 were enrolled at each site. The MESA Stress participants are similar to the full cohort with the exception of fewer persons in the age category of 75-84 years, slightly more men and more participants with some college education. No differences were significant.

\section{Measures}

Allostatic load

Twelve physiological indicators (resting heart rate [rhr], systolic blood pressure, diastolic blood pressure, sex-specific WHR, body mass index, low-density lipoprotein (LDL) cholesterol, high-density lipoprotein (HDL) cholesterol, glucose, total cholesterol, cortisol, norepinephrine and epinephrine) that were collected at the MESA Stress Exam (between exams 3 and 4) were used to construct AL. Participants fasted for at least 12 hours prior to their clinic visit when the blood was drawn. Due to the chronic nature of AL and the large age range of the sample, we decided to include those who were on medications (lipid lowering, hypertension, oral hypoglycaemic, insulin, beta blockers and ACE inhibitors) as high risk since there may have been damage to various bodily systems prior to beginning medication, as have others. ${ }^{15} 16$ Other studies have chosen to disregard medication use and assign participants as high or low risk based on current values. ${ }^{17}$

\section{Health behaviours}

Health behaviours were defined as smoking, drinking alcohol, physical exercise and diet and were reported at exam 1 . We used health behaviours reported at exam 1 and AL at exam 5 , an interim of approximately 5 years, in order to allow for the cumulative nature of AL. Smokers were those who indicated that they currently smoke as opposed to never smoked or smoked formerly. We chose this categorisation because the cardiovascular health of former smokers is known to improve in as little as a year and our interval was 5 years. Alcohol drinkers were those who indicated that they currently drink alcohol as opposed to never drank alcohol or formerly drank alcohol. Data regarding binge drinking was not available.

Physical activity was measured with the MESA Typical Week Physical Activity Survey, a self-report questionnaire adapted from the Cross-Cultural Activity Participation Study, ${ }^{18}$ which asked questions regarding different forms of physical activity such as conditioning, team sports and individual activities. We defined physical activity as reporting 500 or more metabolic equivalent tasks (METs) minutes per week of intentional exercise (eg, walking, dancing, sport and conditioning). A MET expresses the energy cost of a task through the oxygen cost of an activity. ${ }^{19}$ MET minutes are defined by multiplying the number of METs expended in an activity by the number of minutes performed each week. MET levels are determined by the Office of Disease Prevention and Health Promotion, ${ }^{20}$ which recommends at least 500 MET minutes a week of physical activity for all adults, so we chose a 500 MET minute threshold as the cut-off for those who get physical activity versus those who do not.
Table 1 Latent class analysis fit statistics for 2-6 class models in the Multiethnic Study of Atherosclerosis Stress Sub-study, 2004-2005

\begin{tabular}{llllll}
\hline Class \# & AIC & BIC & Entropy & $\begin{array}{l}\text { LMR } \\
\text { ( } p \text { value) }\end{array}$ & $\begin{array}{l}\text { BLRT } \\
\text { ( } p \text { value) }\end{array}$ \\
\hline 2 & 9663.61 & 9756.89 & 0.917 & $749.53(0.000)$ & $749.53(0.000)$ \\
3 & 9598.53 & 9740.91 & 0.800 & $85.08(0.000)$ & $85.08(0.000)$ \\
4 & 9574.80 & 9766.28 & 0.737 & $43.73(0.000)$ & $43.73(0.000)$ \\
\hline 5 & 9571.96 & 9812.53 & 0.776 & $22.84(0.325)$ & $22.84(0.267)$ \\
\hline 6 & 9566.75 & 9855.37 & 0.736 & $25.87(0.250)$ & $26.28(0.102)$ \\
\hline
\end{tabular}

BLRT, bootstrapped likelihood ratio test; LMR, Lo-Mendell-Rubin.

Poor diet was modelled with two empirically derived variables. Diet data were collected by the Food Frequency Questionnaire (FFQ), which provides a list of common foods such as fruits, vegetables, beans, soda and snacks and asks how often a person consumes them. Previous MESA authors, who conducted the ancillary diet study, created food groups based on the FFQ. ${ }^{21}$ We followed the methods of previous MESA diet researchers by conducting principle components analysis and factor analysis and found that four factors best represented the eating habits of the sample. Two factors were considered 'poor diet' (sweets and soda; fats, oils and fried foods), and two factors were 'healthy diet', which was characterised by cheese, fruit, vegetables, legumes and fish. For this analysis, we used only the poor diet factors because poor diet is thought to most affect AL. Those who had factor scores in the top 25th percentile for each poor diet factor were considered to have a poor diet. Covariates were sex, years of education, age and race.

\section{Statistical analysis}

LCA was estimated with 12 dichotomous biological indicators to find clusters of AL indicators. We compared Akaike Information Criterion (AIC), Bayesian Information Criterion (BIC), entropy, Lo-Mendell-Rubin (LMR) and bootstrapped likelihood ratio test (BLR) for models with 2-6 classes $^{22}$ to compare model fit and select the most parsimonious model. Table 1 shows the fit statistics for models with 2-6 classes. We selected the model with four classes based on the BLR and LMR, which indicates that a four-class model fits significantly better than a three-class model. One of the main assumptions of LCA is that after conditioning on the latent variable, ${ }^{23}$ indicators are independent of one another. A violation of this assumption means that variables are related to one another within latent classes, which can result in fit statistics that are too high and more classes than necessary.

In order to check this assumption, we compared observed standardised bivariate residuals to those expected from the model. We found that most residuals were within normal limits, but there were a few that were larger than preferred. In order to correct this, we collapsed some variables that were highly correlated (BMI and WHR; LDL cholesterol and total cholesterol; and norepinephrine and epinephrine) and continued with one variable that represented both (eg, BMI or WHR), and participants were considered as having that indicator if they were in the top $25 \%$ of either variable. We also eventually excluded $\mathrm{rhr}$ as an indicator because $99 \%$ of the sample had an rhr of less than 100 , so it did not discriminate between classes well. The final model used nine indicators-systolic blood pressure, diastolic blood pressure, body mass index, WHR, glucose, cortisol, HDL cholesterol, combination 2 (LDL cholesterol or total cholesterol) and combination 3 (norepinephrine or epinephrine). Participants were 
Table 2 Allostatic load indicators by percentile, sample demographics and health behaviours in the Multiethnic Study of Atherosclerosis Stress Substudy, 2004-2005

\begin{tabular}{|c|c|c|c|}
\hline Variables & 25th percentile & Mean & 75th percentile \\
\hline \multicolumn{4}{|l|}{ Allostatic load } \\
\hline Resting heart rate (bpm) & 57.50 & 64.50 & 71.00 \\
\hline Systolic blood pressure (mm Hg) & 108.50 & 123.40 & 136.00 \\
\hline Diastolic blood pressure (mm Hg) & 63.50 & 69.88 & 76.50 \\
\hline \multicolumn{4}{|l|}{ Waist-to-hip-ratio (in) } \\
\hline Male & 0.93 & 0.98 & 1.02 \\
\hline Female & 0.87 & 0.92 & 0.98 \\
\hline Body mass index $(\%)^{*}$ & 25.20 & 29.04 & 32.0 \\
\hline Low-density lipoprotein cholesterol (mg/dL) & 91.00 & 113.43 & 133.00 \\
\hline High-density lipoprotein cholesterol (mg/dL) & 41.00 & 51.33 & 59.00 \\
\hline Glucose (mg/dL) & 88.00 & 102.68 & 105.00 \\
\hline Total cholesterol (mg/dL) & 166.00 & 190.04 & 211.00 \\
\hline Cortisol (ng/dL) & 5.51 & 12.00 & 21.80 \\
\hline Norepinephrine (ng/dL) & 11.50 & 15.29 & 21.14 \\
\hline Epinephrine (ng/dL) & 0.86 & 1.36 & 2.22 \\
\hline Variables & $\mathbf{n}$ & $\%$ & Total \\
\hline \multicolumn{4}{|l|}{ Demographics } \\
\hline \multicolumn{4}{|l|}{ Age categories (years) } \\
\hline $45-54$ & 170 & 17 & 1002 \\
\hline $55-64$ & 310 & 31 & \\
\hline $65-74$ & 333 & 33 & \\
\hline $75-84$ & 189 & 19 & \\
\hline Female sex & 526 & 53 & \\
\hline \multicolumn{4}{|l|}{ Race } \\
\hline White, Caucasian & 188 & 19 & 1002 \\
\hline Black, African-American & 286 & 29 & \\
\hline Hispanic & 528 & 53 & \\
\hline \multicolumn{4}{|l|}{ Education } \\
\hline High school/GED & 202 & 20 & \\
\hline Bachelor's degree & 111 & 11 & \\
\hline \multicolumn{4}{|l|}{ Medications } \\
\hline Any lipid lowering med & 261 & 27 & 980 \\
\hline Any hypertension meds & 466 & 48 & 980 \\
\hline Oral hypoglycaemic & 125 & 13 & 980 \\
\hline Insulin & 24 & 2.5 & 980 \\
\hline \multicolumn{4}{|l|}{ Health Behaviours } \\
\hline Physical activity (>=500 METs per week) & 587 & 59 & 1002 \\
\hline Smoker (current) & 113 & 11 & 1002 \\
\hline Alcohol (current) & 538 & 54 & 1001 \\
\hline Poor diet: sweets and soda & 269 & 27 & 1002 \\
\hline Poor diet: fats, oils and fried foods & 271 & 27 & 1002 \\
\hline
\end{tabular}

GED, General Education Development; METs, metabolic equivalent tasks.

given a point for each indicator for which their score was in the top $25 \%$ of the sample distribution with the exception of HDL cholesterol, which was for the lower $25 \%$ of the sample distribution.

After the four class model was selected, we fit latent class regression of class on health behaviours. We conducted regressions with individual health behaviours predicting class and a multivariate model that included all health behaviours. All models were adjusted for sex, education, race and age. Descriptive statistics were conducted in Stata V.13, ${ }^{24}$ and all latent variable models were completed in Mplus V.7.1. ${ }^{25}$

\section{RESULTS}

Sample

Table 2 lists the 25th and 75 th percentile for each AL indicator and provides descriptive statistics for the sample. The sample was composed of 1002 participants who were enrolled in the Mesa Stress ancillary study of which 53\% were women, 


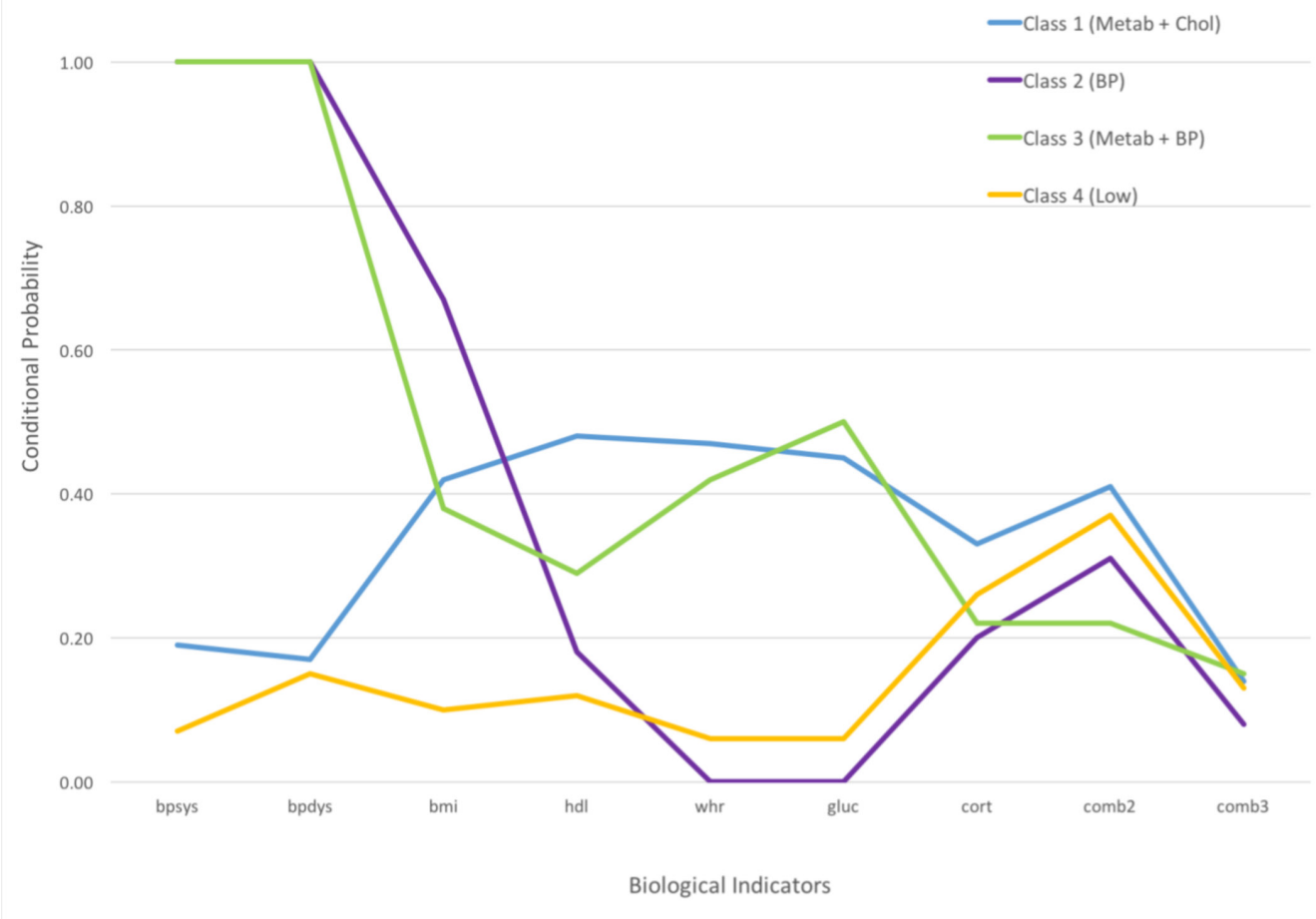

Figure 1 Conditional probabilities ( $y$-axis) for each biological indicator ( $x$-axis) by class. Multi-ethnic Study of Atherosclerosis Stress Sub-study (2004-2005).

$29 \%$ were black and 53\% were Hispanic. Three per cent of the sample was missing cortisol, norepinephrine and epinephrine; however, none were missing more than three indicators out of nine, so we used all of the available data to estimate AL.

\section{AL classes}

Figure 1 shows the conditional probabilities for each indicator by class. We labelled class 1 'Metabolic+Cholesterol' (17\% prevalence), because it featured high rates for WHR, body mass index, HDL cholesterol, glucose and combination 2 (total cholesterol or LDL cholesterol). The second class was labelled 'Blood Pressure' (14\% prevalence), because it featured high rates of systolic blood pressure and diastolic blood pressure.

The third class was labelled 'Metabolic+Blood Pressure' (36\% prevalence), because it included high rates of systolic blood pressure, diastolic blood pressure, WHR, body mass index and glucose. The final class was labelled 'low' (34\% prevalence), because it featured low rates of all physiological indicators.

\section{AL class and health behaviours Unadjusted models}

When class membership was regressed on individual health behaviours, controlling for demographic variables, the 'metabolic+blood pressure' class was the only class that showed significant associations with any of the health behaviours (see table 3). Those having more than 500 MET minutes of exercise per week were significantly less likely to be in the 'metabolic +blood pressure' class compared with the 'low class'. Similarly, those who reported current alcohol use were less likely to be in the 'metabolic+blood pressure class' compared with the 'low class'. Poor diet and smoking were not significant predictors of any of the AL classes.

\section{Adjusted models}

When all of the health behaviours were included in the model, the results were similar to the individual models, with physical

Table 3 ORs and $95 \%$ Cls from latent class regression models of allostatic load class on individual health behaviours

\begin{tabular}{lllll}
\hline & Class 1 'Metabolic+Cholesterol' & Class 2 'Blood Pressure' & Class 3 'Metabolic+BP' & $\begin{array}{l}\text { Class } 4 \text { 'Low' } \\
\text { (reference) }\end{array}$ \\
\hline Physical activity (>=500 METs/week) ${ }^{*}$ & $0.78(0.41$ to 1.47$)$ & $1.73(0.91$ to 3.31$)$ & $0.61(0.41$ to 0.92$)$ & 1.00 \\
Diet_FOF & $1.02(0.50$ to 2.09$)$ & $0.82(0.35$ to 1.26$)$ & $0.75(0.47$ to 1.20$)$ & 1.00 \\
Diet_SS & $1.51(0.33$ to 3.04$)$ & $0.82(0.43$ to 1.56$)$ & $0.85(0.52$ to 1.38$)$ & 1.00 \\
Alcohol (current) & $0.90(0.46$ to 1.75$)$ & $1.82(0.92$ to 3.61$)$ & $0.63(0.41$ to 0.95$)$ & 1.00 \\
Smoking (current) & $2.70(0.76$ to 9.60$)$ & $1.26(0.44$ to 3.57$)$ & $1.74(0.79$ to 3.79$)$ & 1.00 \\
\hline
\end{tabular}

*All models in the table controlled for age, sex, race and education.

Diet_FOF, dietfats, oils and fried foods; diet_SS, diet sweets and soda; METs, metabolic equivalent tasks. 
Table 4 ORs and $95 \%$ Cls from latent class regression models of allostatic load class on health behaviours controlling for other health behaviours

\begin{tabular}{lllll}
\hline & Class 1 'Metabolic+Cholesterol' & Class 2 'Blood Pressure' & Class 3 'Metabolic+BP' & $\begin{array}{l}\text { Class } 4 \text { 'Low' } \\
\text { (reference) }\end{array}$ \\
\hline Physical activity activity $\left(>=500\right.$ METs/week) ${ }^{*}$ & $0.80(0.40$ to 1.59$)$ & $1.77(0.89$ to 3.55$)$ & $0.62(0.42$ to 0.96$)$ & 1.00 \\
Diet_FOF & $0.65(0.28$ to 1.49$)$ & $0.57(0.28$ to 1.16$)$ & $0.75(0.44$ to 1.30$)$ & 1.00 \\
Diet_SS & $2.07(0.90$ to 4.73$)$ & $1.05(0.50$ to 2.21$)$ & $1.09(0.61$ to 1.97$)$ & 1.00 \\
Alcohol (current) & $0.86(0.37$ to 2.01$)$ & $1.86(0.94$ to 3.68$)$ & $0.63(0.40$ to 1.00$)$ & 1.00 \\
\hline Smoking (current) & $3.05(0.75$ to 12.49$)$ & $1.42(0.48$ to 4.16$)$ & $1.86(0.85$ to 6.40$)$ & 1.00 \\
\hline
\end{tabular}

Diet_FOF=DietFats, Oils, Fried Foods.

Diet_SS=DietSweets, Soda.

*All models in the table controlled for age, sex, race, education.

Diet_FOF, dietfats, oils and fried foods; diet_SS, diet sweets and soda; METs, metabolic equivalent tasks.

activity and alcohol use still the only two health behaviours that were significantly associated with class (see table 4 ). The OR for physical activity in class 3 compared with class 4 increased slightly from the individual model and the OR for alcohol use in class 3 compared with class 4 remained the same.

\section{DISCUSSION}

We found that in a multiethnic sample, biological indicators of AL could be separated into four clusters and that the metabolic plus blood pressure class was significantly associated with physical activity and alcohol use. We believe that the results support the idea that a moderate amount of exercise and a low to moderate amount of alcohol can be beneficial to the reduction of metabolic risk factors.

Using LCA, we found four clusters of symptoms within the sample, namely, a metabolic plus cholesterol class, a blood pressure class, a metabolic plus blood pressure class and a low class. Although there are many ways to measure AL, factor and latent measures are gaining popularity. Our metabolic and blood pressure results are consistent with prior research results using factor analysis. ${ }^{26} 27$

Physical activity was related to at least one cluster of AL in our sample. Those who got at least 500 MET minutes of intentional physical activity per week were significantly less likely to be in the metabolic plus blood pressure class than in the low class. Xue et al observed that women who are physically active do not have to engage in time-consuming vigorous exercise to benefit from exercise, but moderate exercise is recommended. ${ }^{28}$ Even those women who had the lowest levels of physical activity had better mortality rates than those who were sedentary. This finding in addition to our findings indicates that moderate exercise, such as walking, done for 2.5 hours per week may be enough to reduce mortality and to confer benefits of reducing cardiovascular risk factors. Although Gay and colleagues found similar results regarding physical exercise and AL, their minimum amount of exercise was substantially higher $(>=1500$ MET minutes per week). ${ }^{5}$ Upchurch et al reported an inverse relationship between $\mathrm{AL}$ and leisure time activity among white, black and Mexican-American women. ${ }^{7}$ They specifically found that participants who reported moderate (600-1500 MET minutes per week) and high (>1500 MET minutes per week) leisure time physical activity had significantly lower AL than those who were inactive. We also conducted the latent class regression with a categorisation o $\mathrm{f}>=1500$ MET minutes per week (results not shown), but no extra benefit was found in our sample.

Those who reported being current alcohol users were also less likely to be in the metabolic plus blood pressure class compared with the low class. Multiple meta-analyses have shown that low-to-moderate alcohol use lowers the risk of metabolic syndrome compared with abstainers and that blood pressure is either positively or neutrally affected by low-to-moderate amounts of alcohol ${ }^{29-31}$ while at least one study has shown higher risk of metabolic syndrome in current drinkers compared with abstainers. ${ }^{32}$ In our study, more than half of the sample reported being current alcohol drinkers but of those who were current drinkers, nearly $60 \%$ reported have $<=2$ drinks a week meaning that the majority of the sample that reported drinking alcohol were low-to-moderate alcohol users. Due to such a large percentage of low-to-moderate drinkers, it is not surprising that alcohol use appeared to confer some benefit in our sample.

Our study had certain limitations. All of health behaviour data were self-reported, so although we had detailed questionnaires regarding physical activity and diet, they were dependent on the respondent's accuracy in reporting. We also did not have specifics as to the type and amount of alcohol that current users drank that might provide a further explanation for the findings regarding alcohol use. Although our sample did have rich biological data, we did not have inflammatory, oxidative stress or telomere markers. These markers have been used to measure physiological dysregulation in the past and have been shown to be sensitive markers of the physical effects of stress. ${ }^{33}$ Future research might also benefit from extending the definition of health behaviour variables beyond diet, exercise, smoking and alcohol use to include variables such as sleep quality, positive and negative affect and social support and isolation.

The strengths of our study include a large multiethnic sample with biological data as well as a statistical approach that allowed for discover of qualitative patterns in AL. Our sample was also

\section{What is already known on this subject}

- The biological systems represented in most measures of allostatic load are independently associated with health behaviours.

- Associations between allostatic load and diet and exercise have been found in very limited populations.

\section{What this study adds}

- We show that a substantially lower amount of physical activity is required to reduce allostatic load compared with previous studies of allostatic load and physical activity.

- We offer further support for the health benefits of low to moderate alcohol use. 
diverse in age, which allowed us to study physical activity in older adults who are typically less active.

In a 2008 commentary by Loucks et al, the authors listed one of the main challenges of employing an AL framework as understanding if AL is affected primarily by psychological stress or if there are other factors that are as or more important such as sedentary lifestyle, smoking or diet. ${ }^{13}$ We addressed this challenge in the current research. Our findings may have practical importance for middle-aged and older adults. For those who have metabolic and blood pressure indicators, moderate exercise such as walking as opposed to more intense exercise may be an inexpensive way to help reduce the risk of cardiovascular factors. Our results may also be of importance to practitioners because we show that 'one size fits all' physical activity recommendations may not be best for people with certain patterns of dysregulation. The qualitative differences in AL among our sample reveal the possibility that two people with a similar quantitative value of AL may not have the same risks or confer the same benefits from certain health behaviours. These findings could help to increase our understanding of how patterns of indicators of AL can differ from person to person and result in different outcomes.

Funding This work was supported by National Institutes of Health (grant number 5TL1TR001454-03).

Competing interests None declared.

Patient consent Not required.

Provenance and peer review Not commissioned; externally peer reviewed.

\section{REFERENCES}

1 McEwen BS, Seeman T. Protective and damaging effects of mediators of stress. Elaborating and testing the concepts of allostasis and allostatic load. Ann N Y Acad Sci 1999;896:30-47.

2 McEwen BS, Gianaros PJ. Stress- and allostasis-induced brain plasticity. Annu Rev Med 2011;62:431-45

3 Athyros VG, Katsiki N, Doumas M, et al. Effect of tobacco smoking and smoking cessation on plasma lipoproteins and associated major cardiovascular risk factors: a narrative review. Curr Med Res Opin 2013;29:1263-74.

4 Mattei J, Bhupathiraju S, Tucker KL. Higher adherence to a diet score based on American Heart Association recommendations is associated with lower odds of allostatic load and metabolic syndrome in puerto rican adults. J Nutr 2013:143:1753-9.

5 Gay JL, Salinas JJ, Buchner DM, et al. Meeting physical activity guidelines is associated with lower allostatic load and inflammation in Mexican Americans. J Immigr Minor Health 2015:17:574-81.

6 Tsatsoulis A, Fountoulakis S. The protective role of exercise on stress system dysregulation and comorbidities. Ann N Y Acad Sci 2006;1083:196-213.

7 Upchurch DM, Rainisch BW, Chyu L. Greater leisure time physical activity is associated with lower allostatic load in white, black, and mexican american midlife women: findings from the national health and nutrition examination survey, 1999 through 2004. Womens Health Issues 2015;25:680-7.

8 Kobrosly RW, van Wijngaarden E, Seplaki CL, et al. Depressive symptoms are associated with allostatic load among community-dwelling older adults. Physiol Behav 2014;123:223-30.

9 Hawkley LC, Lavelle LA, Berntson GG, et al. Mediators of the relationship between socioeconomic status and allostatic load in the Chicago Health, Aging, and Social Relations Study (CHASRS). Psychophysiology 2011;48:1134-45.
10 Hickson DA, Diez Roux AV, Gebreab SY, et al. Social patterning of cumulative biological risk by education and income among African Americans. Am J Public Health 2012;102:1362-9.

11 Yang Y, Kozloski M. Sex differences in age trajectories of physiological dysregulation: inflammation, metabolic syndrome, and allostatic load. J Gerontol A Biol Sci Med Sci 2011;66:493-500.

12 Doamekpor LA, Dinwiddie GY. Allostatic load in foreign-born and US-born blacks: evidence from the 2001-2010 national health and nutrition examination survey. Am J Public Health 2015;105:591-7.

13 Loucks EB, Juster RP, Pruessner JC. Neuroendocrine biomarkers, allostatic load, and the challenge of measurement: a commentary on gersten. Soc Sci Med 2008;66:525-30.

14 Bild DE, Bluemke DA, Burke GL, et al. Multi-ethnic study of atherosclerosis: objectives and design. Am J Epidemiol 2002;156:871-81.

15 Geronimus AT, Hicken M, Keene D, et al. "Weathering" and age patterns of allostatic load scores among blacks and whites in the United States. Am J Public Health 2006;96:826-33.

16 Seeman M, Stein Merkin S, Karlamangla A, et al. Social status and biological dysregulation: the "status syndrome" and allostatic load. Soc Sci Med 2014;118:143-51.

17 Chyu L, Upchurch DM. Racial and ethnic patterns of allostatic load among adult women in the United States: findings from the national health and nutrition examination survey 1999-2004. J Womens Health 2011;20:575-83.

18 Ainsworth BE, Irwin ML, Addy CL, et al. Moderate physical activity patterns of minority women: the cross-cultural activity participation study. J Womens Health Gend Based Med 1999;8:805-13.

19 Jetté M, Sidney K, Blümchen G. Metabolic equivalents (METS) in exercise testing, exercise prescription, and evaluation of functional capacity. Clin Cardiol 1990;13:555-65.

20 U.S. Department of Health and Human Services. Physical activity guidelines for americans: Washington, DC, 2008.

21 Nettleton JA, Steffen LM, Mayer-Davis EJ, et al. Dietary patterns are associated with biochemical markers of inflammation and endothelial activation in the Multi-Ethnic Study of Atherosclerosis (MESA). Am J Clin Nutr 2006;83:1369-79.

22 Nylund K, Bellmore A, Nishina A, et al. Subtypes, severity, and structural stability of peer victimization: what does latent class analysis say? Child Dev 2007;78:1706-22.

23 Hagenaars JM AL, Applied latent class analysis. edn. Cambridge: Cambridge University Press, 2002.

24 StataCorp LP. Stata statistical software: release 13. College Station, Tx: StataCorp LP, 2013.

25 Muthén LK MB. MPlus user's guide. 6th edn: Muthé and Muthén Los Angeles, CA:1198-2011.

26 Buckwalter JG, Castellani B, McEwen B, et al. Allostatic load as a complex clinical construct: a case-based computational modeling approach. Complexity 2016;21:291-306.

27 McCaffery JM, Marsland AL, Strohacker K, et al. Factor structure underlying components of allostatic load. PLoS One 2012;7:e47246.

28 Xue QL, Bandeen-Roche K, Mielenz TJ, et al. Patterns of 12-year change in physical activity levels in community-dwelling older women: can modest levels of physical activity help older women live longer? Am J Epidemio/ 2012;176:534-43.

29 Poli A, Marangoni F, Avogaro A, et al. Moderate alcohol use and health: a consensus document. Nutr Metab Cardiovasc Dis 2013:23:487-504.

30 Movva R, Figueredo VM. Alcohol and the heart: to abstain or not to abstain? Int $J$ Cardiol 2013;164:267-76.

31 Sun K, Ren M, Liu D, et al. Alcohol consumption and risk of metabolic syndrome: a meta-analysis of prospective studies. Clin Nutr 2014;33:596-602.

32 Chen CC, Lin WY, Li Cl, et al. The association of alcohol consumption with metabolic syndrome and its individual components: the Taichung community health study. Nutr Res 2012:32:24-9

33 Belsky DW, Moffitt TE, Cohen AA, et al. Eleven telomere, epigenetic clock, and biomarker-composite quantifications of biological aging: do they measure the same thing? Am J Epidemio/ 2018;187:1220-30. 